\title{
Análisis de decisiones financieras mediante la programación matemática
}

Analysis of financial decisions through mathematical programming

Análise de decisões financeiras usando programação matemática

Freddy Lenin Villarreal Satama

leninv@uhemisferios.edu.ec

Dirección de Investigación, Universidad Hemisferios (Quito-Ecuador) https://orcid.org/0000-0001-7883-1718

Janneth Efigenia Núñez Ribadeneira jenunezr@profesores.uhemisferios.edu.ec Facultad de Ciencias Económicas y Empresariales, Universidad Hemisferios, (Quito-Ecuador) https://orcid.org/0000-0002-3938-3542

\section{Diego Ignacio Montenegro Gálvez} diegom@uhemisferios.edu.ec IDE Business School, Universidad Hemisferios (Quito-Ecuador) https://orcid.org/0000-0002-9760-1181

Santiago Andrés Ullauri Betancourt santiagou@uhemisferios.edu.ec Dirección de Investigación, Universidad Hemisferios (Quito-Ecuador) https://orcid.org/0000-0003-0858-3178

\author{
Geovanny Javier Coloma Braganza \\ gcoloma@estudiantes.uhemisferios.edu.ec \\ Universidad Hemisferios (Quito-Ecuador)
}

\section{RESUMEN}

El presente trabajo tiene el propósito de generar una propuesta de portafolio de inversiones basada en la metodología de la programación lineal con el uso del método simplex con la técnica de modelado de dos fases. Para ello se toman los datos de Forex tabla 5 de inversiones que ayudaron a establecer las seis variables del modelo, cada una representando al tipo de inversión del portafolio. Los resultados indican que luego de maximizar la inversión en la función objetivo su resultado es de USD 67.790 y que posteriormente se puede generar un análisis de sensibilidad que va en función del monto que el inversionista desee arriesgar.

Palabras clave: Optimización matemática; método simplex, portafolio de inversiones.

\begin{abstract}
The present work has the purpose of generating an investment portfolio proposal based on the linear programming methodology with the use of the simplex method with the two-phase modeling technique. For this, the data of Forex table 5 of investments that helped to establish the six variables of the model, each one representing the type of investment of the portfolio, are taken. The results indicate that after maximizing the investment in the objective function, its result is USD 67,790 and that a sensitivity analysis can subsequently be generated based on the amount that the investor wishes to risk.
\end{abstract}

Kaywords: Mathematical optimization; simplex method, investment portfolio.

\section{RESUMO}

O presente trabalho tem como objetivo gerar uma proposta de carteira de investimentos baseada na metodologia de programação linear com a utilização do método simplex com a técnica de modelagem em duas fases. Para isso, são tomados os dados da tabela Forex 5 de investimentos que ajudaram a estabelecer as seis variáveis do modelo, cada uma representando o tipo de investimento da carteira. Os resultados indicam que após maximizar o investimento na função objetivo, seu resultado é de US\$ 67.790 e que uma análise de sensibilidade pode ser gerada posteriormente com base no valor que o investidor deseja arriscar.

Palavras-chave: Otimização matemática; método simplex, carteira de investimentos. 


\section{I.- Introducción}

Las inversiones en la Bolsa de Valores tienen sus orígenes en por el siglo XVI, en Bélgica, en la que se realizaban importantes transacciones económicas, pues se conoce que en el edificio de propiedad de la familia Van Der Buerse disponían de tres monederos de piel en forma de Bolsa cuyos valores recaudados se destinaban para la inversión, por lo que posteriormente se construyó la primera Bolsa de Valores en el año 1602 en la ciudad de Ámsterdam, Holanda (Moreno, 2017). En el Ecuador, la primera Bolsa de Valores tuvo sus orígenes en la ciudad de Guayaquil en 1935. Veinte años más tarde se crea la Comisión Nacional de Valores y se expide en 1964 expide la Ley de compañías y aparece la Corporación Financiera Nacional y para 1969 se faculta a fundar y promover la constitución para crear a la Bolsa de Valores de Quito C.A., y en el 2016 reestablecer su inscripción en el Registro Mercantil, según a los datos que han sido proporcionados por la propia Bolsa de Valores de Quito (BVQ, 2021)

No obstante, a pesar de que las inversiones en bolsa se han dado en el Ecuador desde hace más de 50 años, resulta preponderante recalcar que existen bajos rubros que se han invertido en las Bolsas de Valores tanto en la de Guayaquil como en la de Quito por parte de las personas naturales y empresas, que ya sea por desconocimiento o desconfianza deciden no invertir sus ahorros en el mercado bursátil nacional.

Una de las causas que más han incidido ante esta problemática, está dado en la baja capacidad de ahorro que se tiene por parte de la población, pues se conoce que, gran parte de los ingresos de las familias se destinan al gasto en alimentos, salud y educación por lo que se destinarían pocos recursos al ahorro y a la inversión, así, en este sentido las decisiones financieras en el Ecuador no disponen de una cultura financiera para fortalecer sus finanzas personales y como complemento está el escaso conocimiento que se tiene sobre los portafolios de inversión que son conocidos por un número reducido de empresas.

Adicional a ello, la mala imagen que se ha otorgado a las Bolsas de Valores del país por el caso ISSPOL (Instituto de Seguridad Social de la Policía Nacional) en el que se procesó delitos por falsedad documental lo cual ha sembrado desconfianza en el mercado bursátil ecuatoriano, casas de valores y calificadoras de riesgo de acuerdo con lo señalado por la Fiscalía General del Estado (FGE, 2021). Este escenario ha provocado que en las personas naturales generen mayores dudas sobre la inversión de su dinero y de los rendimientos que podrían obtenerse tanto en el corto, mediano y largo plazo, por lo que sus decisiones financieras pueden enfocarse hacia la implementación de otros proyectos de inversión que no se relacionen con el mercado de valores.

En este contexto, es importante mencionar que Ecuador (como cualquier otro país), se mueve a un cambio de paradigma en el mundo de las finanzas, producto de la revolución digital. No solamente es prioritaria la mejora del servicio y la eficiencia a través de experiencias físicas o digitales como la que realizan las bolsas de valores o bancos tradicionales; sino que, debido al bajo ahorro o la necesidad de soluciones específicas, permitan que las personas o los pequeños negocios tengan un soporte más allá de las transacciones financieras en segmentos locales, haciendo que las inversiones y la gestión sean más rápidas y fáciles. Por ejemplo, Moven es un gestor de finanzas digitales diseñado en torno al consumidor móvil, lo que facilita la vida bancaria a través de una gama de análisis fáciles y divertidos, que abarcan juegos y redes sociales. Square es una organización cuyo conocimiento provino de millones de empresas en mercados emergentes que pierden ventas por no poder aceptar tarjetas de crédito, innovando con tecnología para lectores de tarjetas de crédito que se colocan en los teléfonos celulares para poder subir las ventas e invertir en el crecimiento del negocio (Fisk, 2015). Emprendimientos personales llegan mucho más allá en el plano de la utilización de nuevas tecnologías para pagos financieros y otros, como es el caso CODA Coffee que usan Inteligencia Artificial para medir los parámetros de calidad de los granos de café y Blockchain, un enfoque inviolable de base de datos (pear to pear), para poder hacer pagos en 
tiempo real a sus proveedores de manera $100 \%$ segura, y así, garantizar la reinversión de recursos y sostenibilidad de la cadena de valor (Youngdahl \& Hunsaker, 2020).

Entonces, ¿qué se necesita conocer sobre las diferentes alternativas de inversión para personas naturales o pequeños negocios de acuerdo con el monto invertido? El objetivo del presente estudio es generar un modelo de programación matemática en la colocación de diferentes opciones de inversión cuya función objetivo maximiza el benefício esperado bajo ciertas condiciones.

\section{Inversión y títulos en Bolsa de Valores}

La inversión se ha caracterizado por ser una "cantidad limitada de recursos ya sea en dinero, bienes o especies que se destinan hacia el sector productivo para lograr beneficios dentro de un corto, mediano o largo plazo (Ross et al., 2017, p. 42), es decir en términos generales, mediante la inversión se puede otorgar aportaciones de capital.

No obstante, de acuerdo con la perspectiva de la Bolsa de Valores, una inversión se refiere a la cantidad de dinero que se tiene disponible para adquirir títulos valores de renta fija o de renta variable en el mercado bursátil, con la finalidad de obtener una ganancia o beneficio en el futuro (Bolsa de Valores de El Salvador S.A, 2021), por lo que para ello resulta necesario identificar aquellos títulos valores con los que se permita obtener mayores márgenes de rentabilidad en el futuro, para que posteriormente este tipo de activos sea posible adquirirlos a través de una casa de valores aprobada por la Bolsa de Valores de su territorio.

Por consiguiente, debido a que es indispensable reconocer a los tipos de activos que se pueden adquirir en el mercado bursátil, es necesario clasificarlos y explicarlos a cada uno de ellos por separado tal como se describen en la tabla siguiente:

Tabla 1. Títulos valores de renta fija y de renta variable del mercado bursátil

\begin{tabular}{llll}
\hline \multicolumn{1}{c}{ Tipo } & \multicolumn{1}{c}{ Descripción } & Títulos valores \\
\hline $\begin{array}{l}\text { Renta } \\
\text { fija }\end{array}$ & $\begin{array}{l}\text { Se conforman por títulos valores en los que el emisor está obligado a } \\
\text { pagar cada cierto periodo de tiempo una cantidad de dinero que se ha } \\
\text { señalado con anterioridad }\end{array}$ \\
& $\begin{array}{l}\text { Se conforma por uno o varios activos financieros en que la } \\
\text { Renta } \\
\text { variable }\end{array}$ & $\begin{array}{l}\text { Obligaciones } \\
\text { recuperación del valor invertido y sus rendimientos no están }\end{array}$ & \\
& garantizados.
\end{tabular}

Nota: Adaptado del libro Aplicado para Finanzas Empresariales (Court, 2013, p. 92) y del Artículo Análisis de la Relación entre Rentabilidad y Riesgo en la Planeación de las Finanzas Personales (Villada et al., 2018)

\section{Casas de valores autorizadas para negociar en las Bolsas de Valores del Ecuador}

Las Casas de Valores se caracterizan por ser "entidades de índole privado que han sido autorizadas por la Superintendencia de Compañía, Valores y Seguros para brindar asesoría tanto en la inversión como en financiamiento en el mercado de valores ecuatoriano" (Bolsa de Valores de Quito S.A, 2021), es decir, que si se desea adquirir títulos de inversión en la Bolsa de Valores del país se puede realizar a través de las correspondiente Casas de Valores que están autorizadas por parte de la Superintendencia de Compañías, Valores y Seguros. 
Por lo tanto, las Casas de Valores en la que se pueden adquirir acciones, bonos, obligaciones u otros títulos que se negocian en las Bolsas de Valores del país, se encuentran las siguientes:

Tabla 2. Casas de valores

\begin{tabular}{cccc}
\hline No. & Nombre de la Casa de Valores & No. & Nombre de la Casa de Valores \\
\hline $\mathbf{1}$ & Asesoval Casa de Valores S.A. & 16 & Picaval S.A. \\
\hline $\mathbf{2}$ & Ventura S.A. & 17 & Orion Casa de Valores S. A \\
\hline $\mathbf{3}$ & Vector Global WMG S.A. & 18 & Banrio Casa de Valores S.A. \\
\hline $\mathbf{4}$ & Probrokers S.A. & 19 & Mercapital S.A. \\
\hline $\mathbf{5}$ & Kapital One Kaovalsa S.A. & 20 & Casa Real S.A. \\
\hline $\mathbf{6}$ & Plusvalores S.A. & 21 & Ecuabursáitl S.A. \\
\hline $\mathbf{7}$ & Sucaval S.A. & 22 & Value Casa de Valores S.A. \\
\hline $\mathbf{8}$ & Atlántida Accitlan S.A. & 23 & R\&H Asociados S.A. \\
\hline $\mathbf{9}$ & Analítica Securities S.A. & 24 & Futuro Capital S.A. \\
\hline $\mathbf{1 0}$ & Advfin Casa de Valores S.A. & 25 & Flus Bursátil S.A. \\
\hline $\mathbf{1 1}$ & Silvercross S.A. & 26 & Santa Fe Casa de Valores S.A. \\
\hline $\mathbf{1 2}$ & Merchant Valores S.A. & 27 & Valpacífico S.A. \\
\hline $\mathbf{1 3}$ & Masvalores S.A. & 28 & \\
\hline
\end{tabular}

Como se observa en la tabla 2, existe 29 Casas de Valores en todo el territorio nacional, las mismas que se encuentran legalmente constituidas como Sociedad Anónima (S.A) en la Superintendencia de Compañías, Valores y Seguros, por lo cual una persona natural o cualquier tipo de empresa puede acercarse a cualquiera de estas Casas de Valores para adquirir títulos de inversión ya sea de renta fija o de renta variable.

\section{Los tipos de portafolios en el mercado bursátil}

El portafolio de inversión se conforma por un conjunto de instrumentos financieros preseleccionados con fundamentos sólidos de acuerdo con cada perfil de riesgo u horizonte de inversión (García, 2020, p. 28), por lo cual, se pueden adquirir títulos valores ya sea de renta fija o de renta variable o una mezcla de todos ellos.

Se reconoce además que un portafolio de inversión se relaciona con el perfil de riesgo del inversionista, el mismo que se refiere a la capacidad de asumir pérdidas de acuerdo a los niveles de rentabilidad en base a una inversión (Beltrán et al., 2014, p. 110), de tal manera que entre mayor es el riesgo del portafolio, mayor será la rentabilidad que podría obtenerse, mientras que si por el contrario, el riesgo del portafolio es menor se estarían reduciendo los niveles de rendimiento y por ende, los márgenes de ganancias serían más bajos. Para diferenciar a los tipos de portafolio de 
inversión, se ha clasificado a todos ellos en una tabla en la que se comparan con los títulos a adquirir y el perfil de riesgo del inversionista:

Tabla 3. Tipos de portafolio

\begin{tabular}{llll}
\hline Tipo de portafolio & \multicolumn{2}{l}{ Títulos para adquirir } & Perfil de riesgo \\
\hline Conservador & $\bullet 20 \%$ acciones & $\bullet$ Menor riesgo \\
& $\bullet$ & $\bullet$ Menor rentabilidad \\
\hline Moderado & $\bullet 50 \%$ acciones & $\bullet$ Similar riesgo \\
& $\bullet 50 \%$ bonos & $\bullet$ & Similar rentabilidad \\
\hline Agresivo & $\bullet 100 \%$ acciones & $\bullet$ Mayor riesgo \\
& & $\bullet$ & Mayor rentabilidad \\
\hline
\end{tabular}

De acuerdo con la tabla 3, el inversionista que se caracterice por poseer un portafolio conservador puede disponer de una mayor cantidad de bonos entre títulos de renta fija y de renta variable respectivamente, reconociendo que dentro de estos tipos de portafolio se tendría de un menor riesgo, pero también una menor rentabilidad.

Por su parte, el inversionista moderado dispone de una cantidad algo similar en acciones y bonos tanto en renta fija como en renta variable respectivamente, lo cual se posibilita a obtener un mayor rendimiento que un inversionista conservador pero también se estaría incrementando la posibilidad de riesgo, pues los beneficios que podrían obtenerse de los bonos son más viables de pagarse que los dividendos de las acciones que son se han caracterizado por ser títulos de renta variable y por lo tanto, no se garantiza obtener los ganancias que se esperan de acuerdo a lo que se haya planificado.

En cambio, los inversionistas agresivos tienden a colocar sus mayores inversiones en títulos de renta variable pues con ello se genera un mayor rendimiento a pesar de que con ello, se posibilita a determinar un mayor riesgo para las inversiones pues al comprar acciones que son títulos de renta variable se podría incrementar los niveles de riesgo, aunque se podrían tener mayores beneficios inclusive dentro de un corto plazo.

Por otra parte, los bonos se pagan a una tasa de interés específica, por lo que sus beneficios no pueden alterarse continuamente dada su volatilidad, obteniéndose rendimientos durante cada cierto periodo de tiempo que se haya fijado en el contrato que al ser certificados de renta variable poseen mayores niveles de riesgo.

\section{Estado del arte}

Las inversiones financieras como otros campos del conocimiento han sido tratados por diversos autores aplicando las técnicas de optimización matemática. A continuación, se genera la revisión literaria en varios estudios realizados.

(Koslov et al., 2021), en su trabajo consideran el problema de proyectos de inversión en transporte para un fin específico de logística de red cuyo objetivo es optimizar las inversiones tomando en cuenta recursos como financieros, humanos, infraestructura tecnológica que dan lugar a una productividad técnica como parámetros para establecer planes de transporte con la función de maximización del ingreso el resultado principal es el desarrollo de un modelo de optimización matemática que permite determinar la influencia de factores en la estructura de vehículos.

(Bouillon, 2018) analiza un modelo óptimo de inversiones en activos de alto riesgo utilizando la metodología de programación lineal y un modelo markoviano para la toma de decisiones con datos de acciones de tres empresas tomadas de la Bolsa de Valores de Lima para predecir los precios de los activos que tomando en cuenta los resultados muestran que se deben invertir en función del estado naturaleza que estos se encuentren, estando en mejor posición las 
acciones de Credict-c1y Buenavc1 donde es conveniente invertir, sugiriendo que el modelo aplicado puede ser perfectamente utilizado con un portafolio de inversiones ampliado.

Por otro lado (Chica, 2019) presenta un modelo matemático basado en la programación por metas del método simplex multifase para la selección de alternativas de inversión en Pymes el mismo que permitió identificar la dinamización asignando pesos que sustenten al modelo para la selección de la inversión adecuada en una Pyme como aporte a la teoría de las decisiones para el mejoramiento de los niveles de gestión de los administradores.

María (2015) genera un modelo de aplicación con la metodología de programación matemática para el análisis de un amplio proyecto de portafolio de inversiones que consta de diecisiete proyectos del sector agroindustrial, tomando en cuenta varias restricciones para para la inversión de modo que la función objetivo maximiza el retorno al inversor, permitiendo que el modelo maneje de manera óptima una combinación de variables, considerando la tasa de retorno y tasa de oportunidad para el stakeholder.

(Acuña et al., 2013) genera en su trabajo aplicando la programación lineal para la asignación de carga de trabajo a los proyectos bajo la técnica de optimización matemática con la contrastación de una prueba de hipótesis de Bernoulli, cuyo objetivo fue trabajar en la minimización de la diferencia entre la carga máxima y mínima de trabajo, y a pesar de la utilización del modelo aplicado el resultado principal indica que no es coincidente en el $90 \%$ de las interacciones.

(Peraita, 2017), en su tesis doctoral analiza el riesgo para la valoración de activos financieros mediante un vector de probabilidades a partir de los precios de los activos con la metodología de la optimización matemática función minimización de entropía relativa, cuyo resultado principal es la valoración adecuada de los activos reflejando formas no lineales de la volatilidad del mercado.

(Wu \& Ge, 2012), mediante la programación lineal generan la simulación de inversiones y capacidad instalada midiendo de manera óptima la capacidad instalada de centrales eléctricas para una base racional de toma de decisiones estratégicas de inversión cuyo resultado principal presenta que una segunda planta de la central no puede cumplir la solicitud óptima de inversión y operación.

(Atkinson et al., 2021), analizan mediante la programación de enteros mixtos para la generación de inversiones en infraestructura de plantas de combustibles fósiles con un conjunto de variables cuyo resultado permite tomar la decisión de construir nueva infraestructura cada cierto período en la representación de una red insular reduciendo los tiempos de ejecución de más de dos meses a pocas semanas lo que optimiza el costo.

(Kumar et al, 2021), analizan mediante la obtención de un modelo algorítmico para el manejo de finanzas personales basadas en el análisis predictivo. El modelo guía al usuario inversor la categoría de predicción más conveniente, reflejando los valores potenciales y que puede ayudar a los clientes usuarios a estimar de mejor forma la transacción que realizará para la obtención de un óptimo rendimiento.

(Cruz et al., 2004), en su trabajo exponen el procedimiento para la colocación de inversiones que generen un mayor rendimiento mediante instrumentos financieros de renta fija, simulado en un programa de Excel_Solver, cuyos principales resultados indican la conveniencia de invertir en el primero, tercero y cuarto mes del período y no es recomendable invertir en el segundo mes, prestándose el modelo a varias simulaciones dependiendo de la inversión de las personas.

(Zavala \& García, 2009), muestran la asignación de inversiones en una cartera de máximo rendimiento y mínimo riesgo, basado en el método simplex de programación lineal asignados en la Bolsa de Valores Mexicana cuyos resultados principales observados indican que es posible generar una cartera de inversión minimizando el riesgo financiero en comparación con el cálculo de un 
modelo complementario estadístico que no responde al resultado requerido por lo que resulta útil el manejo del modelamiento matemático al ser más preciso.

Bermúdez (2011) genera un estudio documental informativo, en el que aborda los aportes de la comunidad científica, respecto a temas relacionados con programación lineal, en las que evidencia que los modelos de optimización basados en programación lineal son utilizados en ambientes reales para la solución de problemas y ayuda a la toma de decisiones que contribuyen a la reducción de costos, mejorar la productividad en la planificación de la producción para el aprovechamiento de la capacidad de reducción en inventarios.

(Mallo et al., 2019), examinan el planteamiento metodológico de la programación lineal y la teoría de las probabilidades aleatorias para escenarios de incertidumbre, asignando un grado de pertenencia previa a su aplicación basado en el VAN y TIR de la inversión de proyectos, seleccionando el menor valor de pertenencia del proyecto a cada restricción objetivo, eligiendo en la simulación el cuarto proyecto pues cumple con el menor grado de 0,6 de cumplimiento del objetivo.

En el caso de manejo y gestión de proyectos, la investigación de operaciones es útil como lo señala Terrazas (2012) para la mejor asignación de recursos en actividades competidoras. En el análisis de factibilidad de proyectos una dificultad es saber ubicar un proyecto en las mejores condiciones operativas para minimizar costos y maximizar ganancias.

(Faulín \& Juan, 2016), analizan el uso de la programación lineal y programación lineal entera en varias áreas del conocimiento para la resolución efectiva con la ayuda de hojas de cálculo y programa especializado de investigación operativa Lindo, en la enseñanza de técnicas cuantitativas para la resolución de problemas de economía y empresa, industria textil, agrícola, automotriz, banca, hospitales, entre otros.

Las compañías de retail, realizan la programación de visitas con el diseño de jornadas de horario semanal y secuenciamiento de visitas adaptada a la demanda de clientes basado en la técnica de la programación lineal entera mixta de las compañías SC Johnson, cuyo resultado principal de este enfoque cubren una mayor área de ventas de $68 \%$ a $88 \%$ con el uso de los mismos recursos de personal (Miranda, 2017).

Un importante estudio es el realizado por (Pérez et al., 2019), en el que exponen su trabajo de asignación de clientes hacia asesores comerciales en una empresa de tecnología para satisfacer la demanda de clientes beneficiarios y con ello generar un plan de contratación de nuevos asesores que en total serían 29 de los 35 iniciales que se encontraba planificado, que hablen varios idiomas, para cubrir la demanda insatisfecha del 99,20\% y sus expectativas de la organización, basado en la técnica de la programación lineal entera mixta.

En las plantaciones forestales es importante la asignación de turnos técnicos con el criterio de máxima productividad y máximo valor esperado de la tierra basado en la técnica de programación lineal entera en la determinación de turnos mono criterios, y también utilizando la técnica de programación por metas ponderada y lexicográfica entera en la determinación de turnos múltiples objetivos que variaron entre 31 y 35 como turnos técnicamente óptimos. (Mariel et al., 2019).

\section{III.- Metodología}

En el desarrollo del presente trabajo se ha utilizado una investigación aplicada, la misma que tiene como finalidad "resolver un problema o planteamiento específico enfocándose hacia la búsqueda de conocimiento para su implementación" (Bernal, 2018, p. 95). 
Es decir, en el estudio actual se utiliza la investigación aplicada al analizar cada una de las acciones que se encuentran disponibles, para que mediante un Modelo de Programación Matemática se permita elegir la mejor decisión financiera con la que se permita obtener una mayor rentabilidad para los inversionistas obteniendo, así mayores conocimientos sobre las alternativas de inversión disponibles que es posible identificarlas en el mercado bursátil.

En cuanto a los métodos de investigación, se ha visto necesario que se aplique el método inductivo y deductivo mediante el cual consiste en realizar generalizaciones utilizando observaciones específicas partiendo de hechos particulares para llegar a una conclusión general. Para que posteriormente, tomando en cuenta estas mismas conclusiones sea posible extraer premisas de forma individual, otorgando un conjunto de afirmaciones que se consideran como ciertas (Del Cid, et al., 2017, p. 75).

Bajo esta explicación, en el trabajo actual se aplica el método inductivo al reconocer cada una de las razones en las que las personas naturales se les dificulta tomar decisiones financieras adecuadas para invertir en la Bolsa de Valores de Quito, por lo que se identifican hechos particulares para obtener una conclusión general.

Luego de ello, se ha utilizado el Modelo de Programación Lineal con el cual es posible obtener conocimientos suficientes para que dé un conjunto de acciones disponibles sea posible seleccionar a las que sean más adecuadas para invertir en ellas obteniendo así un máximo rendimiento, por lo que ante este tipo de escenario se parte desde una teoría ya planteada para identificar soluciones o premisas particulares implementando así el método deductivo.

\section{La programación matemática y las decisiones financieras}

La programación matemática se enfoca en el "desarrollo de métodos para la resolución de problemas de optimización condicionada, en el cual se busca la función objetivo que ha sido sometida a un conjunto de restricciones que necesariamente deben cumplirse" (Taha, 2016, p. 86).

Se reconoce que la programa matemática busca resolver problemas tomando en cuenta un conjunto de variables dentro del área de inversión, esto puede darse ya sea por maximización de beneficios o minimización de riesgo con el modelo de Programación Lineal, el cual busca "optimizar la función objetivo, ya sea maximizando u minimizando, planteado en un conjunto de inecuaciones de primer grado (Kong, 2013, p. 33).

En el Modelo de Programación Lineal otorgan posibles soluciones de acuerdo con la función objetivo que se haya planteado, en relación con cada una de las incógnitas identificadas en el problema, por lo cual se plantean inecuaciones que, al graficarse entre sí, se identifican coordenadas con las es posible determinar la función objetivo que puede ser de maximización o de minimización.

De acuerdo con Martínez et al. (2016, p. 37), para resolver un Modelo de Programación Lineal resulta necesario que se realicen un conjunto de etapas consecutivas, entre las cuales se describen destaca el uso del método simplex de dos fases.

\section{El modelo de programación lineal}

Para Taha (2012) la solución al modelo matemático responde a si satisface todas las restricciones y a su vez se optimiza cuando genera el mejor valor posible. La programación lineal parte de la declaración de la función objetivo y las restricciones inherentes a los recursos 
disponibles y que a su vez son escasos. Existen otros métodos disponibles para la solución de problemas como es el método de programación entera, programación dinámica, programación en red, programación no lineal.

Se precisa el algoritmo matemático que obedece a las variables, que nos define el dominio en el que se encuentra la solución óptima, y las restricciones lineales como un conjunto de ecuaciones e inecuaciones que muestran las limitaciones empresariales de recursos, sin embargo, no todos los problemas de optimización matemática pueden ser resueltos con algoritmos, por lo que es necesario acudir a la solución heurística y metaheurística.

\section{Fases del estudio de programación matemática}

Las fases del estudio comprenden varias etapas que se deben cumplir en orden cronológico, partiendo de la definición del problema y el objetivo a seguir para una solución óptima. Es importante que en esta fase se disponga de los datos para en lo posterior generar las variables que permitirá la construcción del modelo matemático y observar si la solución será o no la óptima previo a la implementación. El arte es el modelado y la habilidad para desarrollar el modelo, y la ciencia está en la matemática con juicio técnico. Soot (2018) afirma que sin importar el campo del cual provenga el problema y lo específico del tema abordado, se analiza el proceso con un modelo matemático que contiene las fases de la programación matemática, tal como se indica en la figura 1:

Figura 1. Estructura del proceso de modelación de un problema de optimización

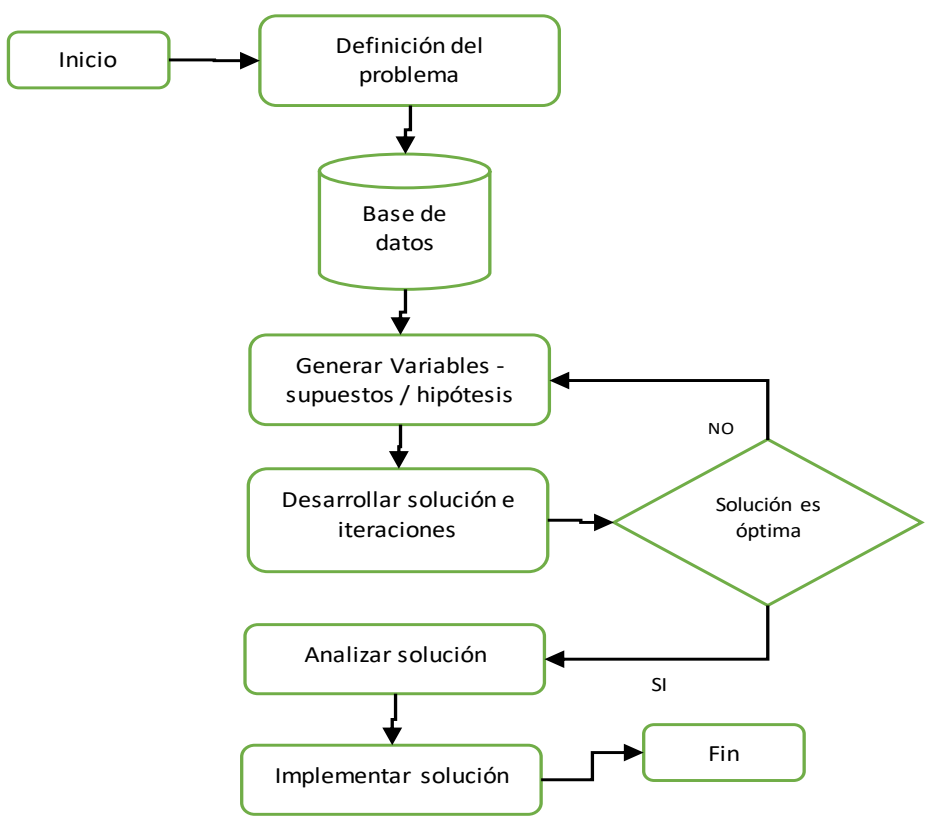

El método Simplex (Izar, 1996). - Es un procedimiento general de programación matemática para llegar a la solución óptima que se obtiene en un número finito de pasos. Estos son:

$\rightarrow$ La solución es cualquier conjunto de variables $X_{j}$ que satisfacen las restricciones del problema $(A x=B)$.

$\rightarrow$ La solución factible es aquella que satisface la no - negatividad de las restricciones $\left(X_{j \geq 0}\right)$. 
$\rightarrow$ La solución básica del sistema de $m$ ecuaciones lineales son $n$ variables $A x=B,(m<n)$, cuyo rango $R(A)=m$ (variables básicas). La solución es $n-m=0$; y, resolviendo para las $m$ variables restantes, cuando el determinante de sus coeficientes no sean cero.

$\rightarrow$ La solución básica factible es tal, que todas las $m$ variables básicas $x_{j} \geq 0$.

$\rightarrow$ La degeneración de una solución básica $(A x=B)$ se da si una o más variables básicas son iguales a cero.

Figura 2. Proceso de metodología Simplex.

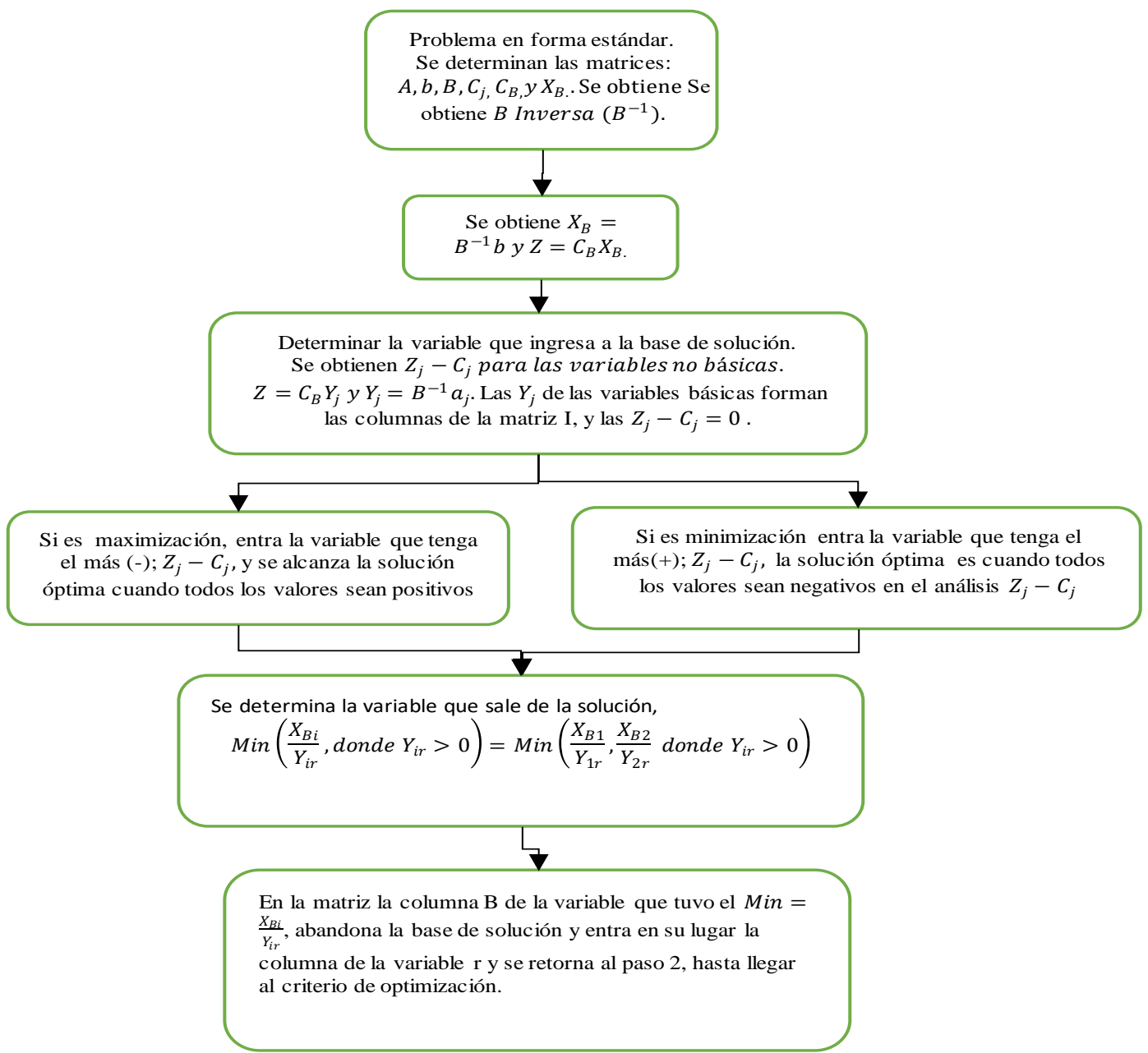

Tabla4. Matriz Método Simplex.

\begin{tabular}{|c|c|c|c|c|c|c|c|}
\hline & & & $c_{j}[$ & $c_{1}$ & $c_{2}$ & $c_{3} \ldots$ & $c_{n}$ \\
\hline$C_{B}$ & $X_{B}$ & $b$ & & $x_{1}$ & $x_{2}$ & $x_{3} \ldots$ & $x_{n}$ \\
\hline & & & $Z^{*}$ & $a_{1}$ & $a_{2}$ & $a_{3} \ldots$ & $a_{n}$ \\
\hline
\end{tabular}

Donde:

$X_{B}=$ Vector de la solución básica factible.

$b=$ variables básicas.

$Z^{*}=$ función objetivo (Max - Min) 
$C_{j}=$ Vector de costos y $C_{B}=$ Vector de componentes $\mathrm{C}$

$Z_{j}=C_{B} X_{B}$ y $Z_{j}-C_{j}=C_{B} X_{B}-C_{j}$

$y_{r j}=$ Nueva solución básica factible

\section{Método de las dos fases}

Este método elimina el uso de la constante $M$, siendo la fase I el proceso por el cual se trata de encontrar la solución básica inicial, y si esta aparece, se invoca ala fase II que dará la solución al problema original donde las variables deben ser reales y mayores a cero, el detalle de estas fases se describe a continuación (Mathstools, 2021):

- Fase I, Añadir $k$ columnas mediante variables artificiales a la matriz $A$, de modo que contenga una matriz identidad de tamaño $m \times n$.

- Cambiar la función objetivo original por ceros a excepción de la última $k$ componentes.

- Generar el algoritmo que puede dar lugar a la solución óptima, en el que las variables artificiales sales de la base para dar paso a la fase II, o puede darse el caso de que el problema arroje una solución óptima finita diferente de cero, por lo que el problema original no tendría solución.

- Fase II, eliminadas las variables artificiales usando la función objetivo original.

- Tomar las m primeras columnas de la matriz A.

- Seguir el algoritmo hasta llegar a las cuatro posibles salidas del problema.

\section{Base de datos y variables}

Tabla 5.- Inversiones

\begin{tabular}{lccc}
\hline \multicolumn{2}{c}{ Inversión } & \multicolumn{2}{c}{$\mathbf{\$ 1 0 0 . 0 0 0}$} \\
\hline Inversión & Interés & \multicolumn{2}{c}{ Inversión máxima } \\
\hline Oro & $35 \%$ & $\$$ & $38.000,00$ \\
\hline Bitcoin & $48 \%$ & $\$$ & $45.000,00$ \\
\hline EUR/USD & $10 \%$ & $\$$ & $10.000,00$ \\
\hline Ethereum & $21 \%$ & $\$$ & $30.000,00$ \\
\hline Gas natural & $69 \%$ & $\$$ & $35.000,00$ \\
\hline Cardano & $12 \%$ & $\$$ & $12.000,00$ \\
\hline Plata & $0,03 \%$ & $\$$ & $3.000,00$ \\
\hline USD/CAD & $0,05 \%$ & $\$$ & $5.500,00$ \\
\hline Dogecoin & $0,09 \%$ & $\$$ & $1.500,00$ \\
\hline
\end{tabular}

Nota: Tomado de Forex Online Trading (2021)

En estadística, se conoce como población al conjunto de elementos que poseen características similares y que son objetivo de un estudio a realizar (Newbold et al., 2016, p. 6), por lo cual en el desarrollo de este trabajo se toma en cuenta los datos de la tabla 4, con aquellas empresas que presentan la información, en el que puede resultar una población realmente pequeña, 
sin embargo, los lectores puedan tener la capacidad de poder ampliar la base de datos. Las variables y su definición la podemos apreciar en la tabla 6.

Tabla 6.- Variables del modelo de inversión

\begin{tabular}{|c|c|c|}
\hline Inversión & Variable & Definición \\
\hline & Función objetivo & Maximizar inversión \\
\hline Oro & $X_{1}$ & Dólares invertidos en Oro \\
\hline Bitcoin & $X_{2}$ & Dólares invertidos en Bitcoin \\
\hline EUR/USD & $X_{a}$ & Dólares invertidos en EUR/USD \\
\hline Ethereum & $X_{4}$ & Dólares invertidos en Ethereum \\
\hline Gas natural & $X_{5}$ & Dólares invertidos en Gas Natural \\
\hline Cardano & $X_{6}$ & Dólares invertidos en Cardano \\
\hline
\end{tabular}

\section{V.- Resultados y discusión}

La función objetivo al ser un problema en el que interesa al beneficiario obtener un mayor rendimiento, será de maximizar la inversión. El problema consta de seis variables caracterizadas por el porcentaje de rendimiento esperado y como consecuencia de ello se forman nueve restricciones. Finalmente, las condiciones de no negatividad de las seis variables que deben ser mayores o iguales a cero de esta forma:

\section{Función objetivo:}

Maximizar: $Z=0,35 X 1+0,48 X 2+0,1 X 3+0,21 X 4+0,69 X 5+0,12$ X6

sujeto a:

$1 \mathrm{X} 1+0 \mathrm{X} 2+0 \mathrm{X} 3+0 \mathrm{X} 4+0 \mathrm{X} 5+0 \mathrm{X} 6 \leq 38000$

$0 X 1+1 X 2+0 X 3+0 X 4+0 X 5+0 X 6 \leq 45000$

$0 X 1+0 X 2+1 X 3+0 X 4+0 X 5+0 X 6 \leq 10000$

$0 \times 1+0 \times 2+0 \times 3+1 X 4+0 \times 5+0 \times 6 \leq 30000$

$0 \mathrm{X} 1+0 \mathrm{X} 2+0 \mathrm{X} 3+0 \mathrm{X} 4+1 \mathrm{X} 5+0 \mathrm{X} 6 \leq 35000$

$0 \times 1+0 \times 2+0 \times 3+0 \times 4+0 \times 5+1 \times 6 \leq 12000$

$-0,30 \times 1+0,70 \times 2-0,30 \times 3-0,30 \times 4+0,70 \times 5-0,30 \times 6 \geq 0$

$0,60 \times 1-0,40 \times 2-0,40 \times 3+0,60 \times 4-0,40 \times 5-0,40 \times 6 \geq 0$

$-0,20 \times 1+0,80 \times 2-0,20 \times 3-0,20 \times 4-0,20 \times 5-0,20 \times 6 \geq 0$

$\mathrm{X} 1, \mathrm{X} 2, \mathrm{X} 3, \mathrm{X} 4, \mathrm{X} 5, \mathrm{X} 6 \geq 0$

Pasamos el problema a la forma estándar, añadiendo variables de exceso, holgura, y artificiales según corresponda.

Como la restricción 1 es del tipo ' $\leq$ ' se agrega la variable de holgura X7.

Como la restricción 2 es del tipo ' $\leq$ ' se agrega la variable de holgura X8. 
Como la restricción 3 es del tipo ' $\leq$ ' se agrega la variable de holgura X9.

Como la restricción 4 es del tipo ' $\leq$ ' se agrega la variable de holgura X10.

Como la restricción 5 es del tipo $\leq$ ' se agrega la variable de holgura X11.

Como la restricción 6 es del tipo ' $\leq$ ' se agrega la variable de holgura X12.

Como la restricción 7 es del tipo ' $\geq$ ', y el término independiente negativo o nulo (la restricción se multiplica por -1), se agrega la variable de holgura X13.

Como la restricción 8 es del tipo ' $\geq$ ', y el término independiente negativo o nulo (la restricción se multiplica por -1), se agrega la variable de holgura X14.

Como la restricción 9 es del tipo ' $\geq$ ', y el término independiente negativo o nulo (la restricción se multiplica por -1), se agrega la variable de holgura X15.

MAXIMIZAR: $\mathrm{Z}=0.35 \mathrm{X} 1+0.48 \mathrm{X} 2+0.1 \mathrm{X} 3+0.21 \mathrm{X} 4+0.69 \mathrm{X} 5+0.12 \mathrm{X} 6+0 \mathrm{X} 7+0 \mathrm{X} 8+0 \mathrm{X} 9+0$ $\mathrm{X} 10+0 \mathrm{X} 11+0 \mathrm{X} 12+0 \mathrm{X} 13+0 \mathrm{X} 14+0 \mathrm{X} 15$

sujeto a:
$1 \mathrm{X} 1+1 \mathrm{X} 7=38000$
$0 \mathrm{X} 1+1 \mathrm{X} 2+1 \mathrm{X} 8=45000$
$0 \mathrm{X} 1+1 \mathrm{X} 3+1 \mathrm{X} 9=10000$
$0 \times 1+1 X 4+1 \times 10=30000$
$0 \mathrm{X} 1+1 \mathrm{X} 5+1 \mathrm{X} 11=35000$
$0 \times 1+1 \times 6+1 \times 12=12000$
$0.3 \mathrm{X} 1-0.7 \mathrm{X} 2+0.3 \mathrm{X} 3+0.3 \mathrm{X} 4-0.7 \mathrm{X} 5+0.3 \mathrm{X} 6+1 \mathrm{X} 13=0$
$-0.6 \mathrm{X} 1+0.4 \mathrm{X} 2+0.4 \mathrm{X} 3-0.6 \mathrm{X} 4+0.4 \mathrm{X} 5+0.4 \mathrm{X} 6+1 \mathrm{X} 14=0$
$0.2 \mathrm{X} 1-0.8 \mathrm{X} 2+0.2 \mathrm{X} 3+0.2 \mathrm{X} 4+0.2 \mathrm{X} 5+0.2 \mathrm{X} 6+1 \mathrm{X} 15=0$

$\mathrm{X} 1, \mathrm{X} 2, \mathrm{X} 3, \mathrm{X} 4, \mathrm{X} 5, \mathrm{X6}, \mathrm{X} 7, \mathrm{X} 8, \mathrm{X} 9, \mathrm{X10}, \mathrm{X11}, \mathrm{X} 12, \mathrm{X} 13, \mathrm{X} 14, \mathrm{X} 15 \geq 0$

Con ello se obtienen los valores generados en las interacciones del modelo de programación simplex de dos fases, donde se presentan las variables que entran y salen en cada uno de los pasos que debe cumplir el proceso analizado en PHP Simplex.

Tabla 7.- La variable que sale de la base es P15 y la que entra es P5.

\begin{tabular}{|c|c|c|c|c|c|c|c|c|c|c|c|c|c|c|c|c|c|}
\hline Tabla 1 & & & 0.35 & 0.48 & 0.1 & 0.21 & 0.69 & 0.12 & 0 & 0 & 0 & 0 & 0 & 0 & 0 & 0 & 0 \\
\hline Base & $\mathrm{Cb}$ & P0 & P1 & P2 & P3 & P4 & P5 & P6 & P7 & P8 & $\mathbf{P}$ & P10 & P11 & P12 & P13 & P14 & P15 \\
\hline P7 & 0 & 38000 & 1 & 0 & 0 & 0 & 0 & 0 & 1 & 0 & & 0 & 0 & 0 & 0 & 0 & 0 \\
\hline P8 & 0 & 45000 & 0 & 1 & 0 & 0 & 0 & 0 & 0 & 1 & & 0 & 0 & 0 & 0 & 0 & 0 \\
\hline P9 & 0 & 10000 & 0 & 0 & 1 & 0 & 0 & 0 & 0 & 0 & & 0 & 0 & 0 & 0 & 0 & 0 \\
\hline P10 & 0 & 30000 & 0 & 0 & 0 & 1 & 0 & 0 & 0 & 0 & & 1 & 0 & 0 & 0 & 0 & 0 \\
\hline P11 & 0 & 35000 & 0 & 0 & 0 & 0 & 1 & 0 & 0 & 0 & & 0 & 1 & 0 & 0 & 0 & 0 \\
\hline P12 & 0 & 12000 & 0 & 0 & 0 & 0 & 0 & 1 & 0 & 0 & & 0 & 0 & 1 & 0 & 0 & 0 \\
\hline P13 & 0 & 0 & 0.3 & -0.7 & 0.3 & 0.3 & -0.7 & 0.3 & 0 & 0 & & 0 & 0 & 0 & 1 & 0 & 0 \\
\hline P14 & 0 & 0 & -0.6 & 0.4 & 0.4 & -0.6 & 0.4 & 0.4 & 0 & 0 & & 0 & 0 & 0 & 0 & 1 & 0 \\
\hline P15 & 0 & 0 & 0.2 & -0.8 & 0.2 & 0.2 & 0.2 & 0.2 & 0 & 0 & & 0 & 0 & 0 & 0 & 0 & 1 \\
\hline $\mathbf{Z}$ & & 0 & - & - & - & - & - & - & 0 & 0 & & 0 & 0 & 0 & 0 & 0 & 0 \\
\hline
\end{tabular}


La variable que sale de la base es P14 y la que entra es P2.

Tabla 8.- La variable que sale de la base es P15 y la que entra es P5.

\begin{tabular}{|c|c|c|c|c|c|c|c|c|c|c|c|c|c|c|c|c|c|}
\hline Tabla & & & 0.35 & 0.48 & 0.1 & 0.21 & 0.69 & 0.12 & 0 & 0 & O & $\mathbf{0}$ & 0 & 0 & $\mathbf{0}$ & O & 0 \\
\hline Base & $\mathbf{C b}$ & P0 & P1 & P2 & P3 & P4 & P5 & P6 & P7 & P8 & P9 & P10 & P11 & P12 & P13 & P14 & P15 \\
\hline P7 & 0 & 38000 & 1 & 0 & 0 & 0 & 0 & 0 & 1 & 0 & 0 & 0 & 0 & 0 & 0 & 0 & 0 \\
\hline P8 & 0 & 45000 & 0 & 1 & 0 & 0 & 0 & 0 & 0 & 1 & 0 & 0 & 0 & 0 & 0 & 0 & 0 \\
\hline P9 & 0 & 10000 & 0 & 0 & 1 & 0 & 0 & 0 & 0 & 0 & 1 & 0 & 0 & 0 & 0 & 0 & 0 \\
\hline P10 & 0 & 30000 & 0 & 0 & 0 & 1 & 0 & 0 & 0 & 0 & 0 & 1 & 0 & 0 & 0 & 0 & 0 \\
\hline P11 & 0 & 35000 & -1 & 4 & -1 & -1 & 0 & -1 & 0 & 0 & 0 & 0 & 1 & 0 & 0 & 0 & -5 \\
\hline P12 & 0 & 12000 & 0 & 0 & 0 & 0 & 0 & 1 & 0 & 0 & 0 & 0 & 0 & 1 & 0 & 0 & 0 \\
\hline P13 & 0 & 0 & 1 & -3.5 & 1 & 1 & 0 & 1 & 0 & 0 & 0 & 0 & 0 & 0 & 1 & 0 & 3.5 \\
\hline P14 & 0 & 0 & -1 & 2 & 0 & -1 & 0 & 0 & 0 & 0 & 0 & 0 & 0 & 0 & 0 & 1 & -2 \\
\hline P5 & 0.69 & 0 & 1 & -4 & 1 & 1 & 1 & 1 & 0 & 0 & 0 & 0 & 0 & 0 & 0 & 0 & 5 \\
\hline $\mathbf{Z}$ & & 0 & 0.34 & - & 0.59 & 0.48 & 0 & 0.57 & 0 & 0 & 0 & 0 & 0 & 0 & 0 & 0 & 3.45 \\
\hline
\end{tabular}

Tabla 9.- La variable que sale de la base es P11 y la que entra es P1.

\begin{tabular}{|c|c|c|c|c|c|c|c|c|c|c|c|c|c|c|c|c|c|}
\hline Tabla 3 & & & 0.35 & 0.48 & 0.1 & 0.21 & 0.69 & 0.12 & $\mathbf{0}$ & 0 & 0 & 0 & O & O & 0 & 0 & O \\
\hline Base & Cb & P0 & P1 & P2 & P3 & P4 & P5 & P6 & P7 & P8 & P9 & P10 & P11 & P12 & P13 & P14 & P15 \\
\hline P7 & 0 & 38000 & 1 & 0 & 0 & 0 & 0 & 0 & 1 & 0 & 0 & 0 & 0 & 0 & 0 & 0 & 0 \\
\hline P8 & 0 & 45000 & 0.5 & 0 & 0 & 0.5 & 0 & 0 & 0 & 1 & 0 & 0 & 0 & 0 & 0 & -0.5 & 1 \\
\hline P9 & 0 & 10000 & 0 & 0 & 1 & 0 & 0 & 0 & 0 & 0 & 1 & 0 & 0 & 0 & 0 & 0 & 0 \\
\hline P10 & 0 & 30000 & 0 & 0 & 0 & 1 & 0 & 0 & 0 & 0 & 0 & 1 & 0 & 0 & 0 & 0 & 0 \\
\hline P11 & 0 & 35000 & 1 & 0 & -1 & 1 & 0 & -1 & 0 & 0 & 0 & 0 & 1 & 0 & 0 & -2 & -1 \\
\hline P12 & 0 & 12000 & 0 & 0 & 0 & 0 & 0 & 1 & 0 & 0 & 0 & 0 & 0 & 1 & 0 & 0 & 0 \\
\hline P13 & 0 & 0 & -0.75 & 0 & 1 & -0.75 & 0 & 1 & 0 & 0 & 0 & 0 & 0 & 0 & 1 & 1.75 & 0 \\
\hline P2 & 0.48 & 0 & -0.5 & 1 & 0 & -0.5 & 0 & 0 & 0 & 0 & 0 & 0 & 0 & 0 & 0 & 0.5 & -1 \\
\hline P5 & 0.69 & 0 & -1 & 0 & 1 & -1 & 1 & 1 & 0 & 0 & 0 & 0 & 0 & 0 & 0 & 2 & 1 \\
\hline $\mathbf{Z}$ & & 0 & -1.28 & 0 & 0.59 & -1.14 & 0 & 0.57 & 0 & 0 & 0 & 0 & 0 & 0 & 0 & 1.62 & 0.21 \\
\hline
\end{tabular}

Tabla 10.- La variable que sale de la base es P7 y la que entra es P15.

\begin{tabular}{ccccccccccccccccccc}
\hline $\begin{array}{c}\text { Tabla } \\
4\end{array}$ & & & $\mathbf{0 . 3 5}$ & $\mathbf{0 . 4 8}$ & $\mathbf{0 . 1}$ & $\mathbf{0 . 2 1}$ & $\mathbf{0 . 6 9}$ & $\mathbf{0 . 1 2}$ & $\mathbf{0}$ & $\mathbf{0}$ & $\mathbf{0}$ & $\mathbf{0}$ & $\mathbf{0}$ & $\mathbf{0}$ & $\mathbf{0}$ & $\mathbf{0}$ & $\mathbf{0}$ \\
\hline Base & $\mathbf{C b}$ & $\mathbf{P 0}$ & $\mathbf{P 1}$ & $\mathbf{P 2}$ & $\mathbf{P 3}$ & $\mathbf{P 4}$ & $\mathbf{P 5}$ & $\mathbf{P 6}$ & $\mathbf{P 7}$ & $\mathbf{P 8}$ & $\mathbf{P 9}$ & $\mathbf{P 1 0}$ & $\mathbf{P 1 1}$ & $\mathbf{P 1 2}$ & $\mathbf{P 1 3}$ & $\mathbf{P 1 4}$ & $\mathbf{P 1 5}$ \\
\hline $\mathbf{P 7}$ & 0 & 3000 & 0 & 0 & 1 & -1 & 0 & 1 & 1 & 0 & 0 & 0 & -1 & 0 & 0 & 2 & 1 \\
\hline $\mathbf{P 8}$ & 0 & 27500 & 0 & 0 & 0.5 & 0 & 0 & 0.5 & 0 & 1 & 0 & 0 & -0.5 & 0 & 0 & 0.5 & 1.5 \\
\hline P9 & 0 & 10000 & 0 & 0 & 1 & 0 & 0 & 0 & 0 & 0 & 1 & 0 & 0 & 0 & 0 & 0 & 0 \\
\hline P10 & 0 & 30000 & 0 & 0 & 0 & 1 & 0 & 0 & 0 & 0 & 0 & 1 & 0 & 0 & 0 & 0 & 0
\end{tabular}




\begin{tabular}{|c|c|c|c|c|c|c|c|c|c|c|c|c|c|c|c|c|c|}
\hline P1 & 0.35 & 35000 & 1 & 0 & -1 & 1 & 0 & -1 & 0 & 0 & 0 & 0 & 1 & 0 & 0 & -2 & -1 \\
\hline P12 & 0 & 12000 & 0 & 0 & 0 & 0 & 0 & 1 & 0 & 0 & 0 & 0 & 0 & 1 & 0 & 0 & 0 \\
\hline P13 & 0 & 26250 & 0 & 0 & 0.25 & 0 & 0 & 0.25 & 0 & 0 & 0 & 0 & 0.75 & 0 & 1 & 0.25 & $\begin{array}{c}- \\
0.75\end{array}$ \\
\hline $\mathbf{P 2}$ & 0.48 & 17500 & 0 & 1 & -0.5 & 0 & 0 & -0.5 & 0 & 0 & 0 & 0 & 0.5 & 0 & 0 & -0.5 & -1.5 \\
\hline P5 & 0.69 & 35000 & 0 & 0 & 0 & 0 & 1 & 0 & 0 & 0 & 0 & 0 & 1 & 0 & 0 & 0 & 0 \\
\hline $\mathbf{Z}$ & & 44800 & 0 & 0 & $\begin{array}{c}- \\
0.69\end{array}$ & 0.14 & 0 & $\begin{array}{c}- \\
0.71\end{array}$ & 0 & 0 & 0 & 0 & 1.28 & 0 & 0 & $\begin{array}{c} \\
0.94\end{array}$ & $\begin{array}{c}- \\
1.07\end{array}$ \\
\hline
\end{tabular}

El resultado final de las interacciones expresa que la solución óptima de maximización es de \$67790 con la asignación de inversiones de montos en las siguientes variables:

Tabla 11.- Solución del modelo PL

\begin{tabular}{lcc}
\hline Función objetivo & Variable & Definición \\
\hline $\mathbf{Z}=\mathbf{6 7 . 7 9 0}$ USD & Función objetivo & Maximizar inversión \\
\hline Oro & $X_{1}$ & 38.000 USD en Oro \\
\hline Bitcoin & $X_{2}$ & 45.000 USD en Bitcoin \\
\hline EUR/USD & $X_{3}$ & 10.000 USD en EUR/USD \\
\hline Ethereum & $X_{4}$ & 30.000 USD en Ethereum \\
\hline Gas natural & $X_{5}$ & 35.000 USD en Gas Natural \\
\hline Cardano & $X_{6}$ & 12.000 USD en Cardano
\end{tabular}

\section{VI.- Conclusiones}

En el nuevo paradigma que actualmente vive el mundo, lanzar nuevos proyectos requieren de una inversión meditada de los recursos maximizando la eficiencia. El concepto de emprendimiento de las personas es una actividad fundamental del liderazgo para generar cambio a través de nuevas ideas e iniciativas en un entorno hiperconectado, de aceleración tecnológica y con 4.000 millones de nuevos ciudadanos (Martínez-Barea, 2014); por lo tanto, es crítico elegir la mejor decisión financiera con la que se permita obtener una mayor rentabilidad para los inversionistas obteniendo e identificando las alternativas de inversión disponibles.

En estos días, construir un buen servicio ya no es suficiente para marcar la diferencia. La diferencia que buscan personas e inversores está en la simplicidad para facilitar los procesos y la comprensión del camino más beneficioso para lograrlo. No se habla solamente de "satisfacción del consumidor", sino del "éxito del consumidor" (Peters, 2004). Indiscutiblemente, las decisiones financieras suelen ser algo complejas cuando una persona trata de invertir en la Bolsa de Valores, pues existen diversos instrumentos tanto de renta fija como de renta variable, ya que ante un desconocimiento de ello una gran cantidad de personas no se inclinan por este tipo de inversión. En consecuencia, el entendimiento de estas herramientas es lo que se tiene que lograr a través de este estudio.

El universo poblacional se conforma por aquellas empresas que registran un mayor movimiento en el mercado de inversiones por todas lo que no es posible considerar a todas las 
compañías en el desarrollo del estudio actual. Al utilizar un Modelo de Programación Lineal para la toma de decisiones en un portafolio de inversiones, debemos tomar en cuenta los tiempo y riesgos inherentes al modelo, Para el caso expuesto de varias interacciones la solución con la función objetivo de maximización de inversión indica que el beneficio es de $\$ 67.790$ y que toma en cuenta la asignación de inversión de $X_{1}$ a $X_{6}$ con los valores respectivos mostrados en la tabla 11., por lo que a partir de la primera solución básica factible se pueden generar los escenarios que el investigador vea necesario, llamados análisis de sensibilidad del modelo de programación lineal y que están sujetos al monto inicial que el inversor esté dispuesto a negociar en el portafolio. El modelo analizado puede servir en la aplicación de un portafolio con mayor número de cartera de inversiones tal como lo manifiesta (Bouillon, 2018) en la implementación de una cartera ampliada tomando en cuenta el riesgo y (Chica, 2019) en cuanto a la dinamización de inversiones donde el administrador tiene la oportunidad de mejorar el nivel de gestión para una adecuada toma de decisiones.

Del mismo modo que el trabajo desarrollado por (Kumar et al., 2021), el algoritmo del modelo simplex de dos fases es importante para con los resultados presentados convertir en un esquema de asesoramiento de inversiones en el manejo de finanzas personales de individuos que necesitan esta herramienta basada en el análisis predictivo y de sensibilidad para que los clientes posean información técnica a la hora de elegir inversiones seguras.

La presente investigación tiene como premisas teórico-metodológicas el tipo de Programación que en este caso es Lineal, y la estructura del análisis en dos fases, sin embargo, se podría en posteriores investigaciones aplicar otros métodos matemáticos no lineales que sean adecuados al estudio de otras metodologías de aplicación en la optimización de portafolios de inversiones, sin embargo, de este estudio se pueden derivar la utilización de otras técnicas como la aplicación de procesos markovianos, la utilización de la teoría de Bayes, basada en probabilidades condicionales, métodos probabilísticas tomando en cuenta estados naturaleza, metodologías alternativas como la programación de enteros mixtos y métodos matemáticos en ecuaciones diferenciales muy utilizado en diversas ramas del conocimiento, que pueden orientar al análisis de la conveniencia para el inversor en relación a adquirir títulos valores de renta fija o renta variable, definiendo en qué porcentaje se deberían distribuir esas rentas de tal manera de no afectar a la maximización de las ganancias.

\section{Referencias}

Acuña, S., Madiedo, E., \& Ortíz, N. (Junio de 2013). Modelo de programación lineal binaria para el balance de carga de trabajo en el problema de asignación de proyectos. Ingeniería y Universidad, 17, 167-181.

Arias, J., Bautista, D., \& Meneses, C. (2019). Revisión de literatura sobre los modelos de optimización en programación de turnos de enfermería. UIS Ingenierías, 18(2), 245-258.

Atkinson, T., Preckel, P., \& Gotham, D. (2021). A mathematical programming formulation for long-term infrastructure investment planning in Small Island Developing States. MethosX, 1-8.

Beltrán, E., Campuzano, A., \& Mariño, T. (2014). El mercado alternativo bursátil 2da ed. Barcelona - España: Aranzadi.

Bermúdez, Y. (7 de Julio de 2011). Aplicaciones de programación lineal, entera y mixta. Ingeniería industral, actualidad y nuevas tendencias, 2(7), 85-104

Bernal, C. (2018). Metodología de la Investigación 4ta ed. Administración, economía, humanidades y ciencias sociales. Bogotá Colombia: Pearson.

Bolsa de Valores de El Salvador S.A. (12 de octubre de 2021). Qué es la Inversión en Bolsa. Obtenido de Inversión a través de la Bolsa de Valores: https://www.bolsadevalores.com.sv/index.php/preguntas-inversion-a-traves-de-la-bolsa

Bolsa de Valores de Quito S.A. (20 de octubre de 2021). ¿Qué son las Casas de Valores? Obtenido de https://www.bolsadequito.com/index.php/casas-de-valores-2

BVQ. (30 de octubre de 2021). Historia Institucional. Obtenido de Bolsa de Valores de Quito: https://www.bolsadequito.com/index.php/historia-institucional2

Cavada, J., Cortés, C., \& Rey, P. (28 de Septiembre de 2012). Modelo de planificación y asignación de personal para el patio de equipajes de un aeropuerto internacional. Claio SBPO, 2033-2045. 
Court, E. (2013). Aplicaciones para Finanzas Empresariales. México: Pearson Educación.

Cruz, E., Duarte, T., \& Arias, L. (26 de Diciembre de 2004). Procedimiento Para Realizar Inversiones En Instrumentos Financieros Utilizando La Programación Lineal. Scientia Et Technica, 111-114.

De la Fuente, D., Lozano, J., \& Isabel, F. (1998). Asignación de turnos mediante metaehurísticas, aplicación en empresas asturianas. Revista Asturiana de Economía, 12, 275-287.

Del Cid, A., Méndez, R., \& Sandoval, F. (2017). Investigación: Fundamentos y Metodología. México: Pearson Educación.

Faulín, J., \& Juan, A. (2016). Academia. Recuperado el 3 de Diciembre de 2020, de Aplicaciones de la programación lineal: https://www.academia.edu/download/56760818/Aplicaciones_PL.pdf

FGE. (09 de septiembre de 2021). Caso Isspol: Fiscalía vincula a 6 personas más a la investigación por peculado. Obtenido de Fiscalía General del Estado: https://www.fiscalia.gob.ec/caso-isspol-fiscalia-vincula-a-6-personas-mas-a-lainvestigacion-por-peculado/

Fisk, P. (2015). Gamechangers. ¿Are you Ready to Change the World? Sussex: John Wiley and Sons.

Forex Online Trading. (19 de Diciembre de 2021). Trader.iforex.com. Obtenido de Forex Online Trading: https://trader.iforex.com/webpl3/webtrader.html?view=2

García, M. (2020). Cómo invertir en Bolsa: Aprende todo lo que necesitas saber sobre los mercados bursátiles para ganar dinero a través de estrategias fáciles y rentables a largo plazo. Madrid - España: Kindle.

Izar, J. (1996). Fundamentos de investigación de operaciones. En Fundamentos de investigación de operaciones para administraciòn (págs. 63-88). San Luis Potosí: Universitaria Potosina.

Kong, M. (2013). Investigación de Operaciones: Programación LIneal, Problemas de Transporte y Análisis de Redes. Lima Perú: Fondo Editorial Pontificia Universidad Católica del Perú.

Kumar, M., Chakravarthy, D., Ranganatham, T., \& Ramana, K. (21 de Enero de 2021). Personal finance transaction index scoring using machine learning model. Materials Today: Proceedings, Obtenido de https://doi.org/10.1016/j.matpr.2020.12.235

León, M., Díaz, R., \& Contreras, O. (2007). Utilización del modelo de transporte para la asignación de trabajos a máquinas considerando prioridades. Ingeniería, 11(2), 47-65.

Mallo, P. E., Artola, M., \& Morettini, M. (10 de Octubre de 2019). Decisiones financieras con programación lineal: diferentes estados de la naturaleza. Repositorio digital de la FCE y S-UNMDP, 1-11.

Mariel, O., Aleixo, J., \& Alvares, D. (3 de Mayo de 2019). Modelos matemáticos para la determinación del turno óptimo en plantaciones forestales. Madera y Bosques, 25(1), 1-19.

Martínez, I., Vertiz, G., López, J., Jiménez, G., \& Moncayo, L. (2016). Investigación de Operaciones. México D.F.: Grupo Editorial Patria S.A.

Martínez-Barea, J. (2014). El Mundo que Viene. Barcelona: Gestión 2000.

Mathstools. (5 de Agosto de 2021). mathstools.com. Recuperado el 6 de agosto de 2021, de https://www.mathstools.com/section/main/Metodo_de_las_Dos_Fases?lang=es\#.YQyj2IhKg2x

Miranda, J. P. (Septiembre de 2017). Programación entera para el diseño de jornadas laborales de reponedores en la industria de retail. Ingeniería de sistemas, XXXI, 65-79.

Moreno, J. (10 de noviembre de 2017). La Bolsa de Valores de México durante el porfiriato y la revolución, Obtenido de Revista de Investigación: América Latina en la Historia Económica: https://www.redalyc.org/pdf/2791/279149151004.pdf

Newbold, P., Carlson, W., \& Thorne, B. (2016). Estadística para Administración y Economía 10ma ed. Madrid - España: Pearson Educación.

Ojeda, R. (Junio de 2015). Toma de Decisiones en la Asignación de Personal a Horarios de Trabajo Empleando Programación Lineal: El Caso de una Empresa de Atención Telefónica en México. Investigación administrativa, 44.

Peraita, O. (2017). Valoración de activos financieros por entropía máxima con programación lineal. Madris: Universidad Complutense de Madrid.

Pérez, W., Navarro, N., \& Rocha, J. (22 de Julio de 2019). Modelo de Planeación y Asignación de Personal en la Industria de Servicios con Programación Entera Mixta. Easychair, 1-7.

Peters, T. (2004). ¡Re-imagine! London: Dorling Kindersley Limited.

Ramírez, C., JIménez, Á., \& Garrido, J. (20 de marzo de 2013). Inversión Colombiana en el Exterior, Bavaria y su Estrategia Internacional con las Cervezas. Obtenido de Redalyc: Estudios Gerenciales: https://www.redalyc.org/pdf/212/21208606.pdf

Render, B., Stair, R., \& Hanna, M. (2012). Métodos cuantitativos para los negocios. Ciudad de México: Pearson.

Ross, S., Westerfield, R., \& Jordan, B. (2017). Fundamentos de Finanzas Corporativas 12 ed. México D.F.: Mc Graw Hill Educación.

Soot, T. (2018). Matemáticas Aplicadas a los negocios, las Ciencias Sociales y de la Vida. Toluca: Cengage Learning.

Taha, H. (2012). Investigación de operaciones. Ciudad de México: Pearson. 
Taha, H. (2016). Investigación de Operaciones 11va ed. México D.F: Pearson Educación.

Terrazas, R. (5 de Septiembre de 2012). Aplicación de la programación matemática a la localización de proyectos. Perspectivas, 29, 71-94. Obtenido de http://www.scielo.org.bo/pdf/rp/n29/n29a04.pdf

Villada, F., López, J., \& Múñoz, N. (2018, diciembre 25). Análisis de la Relación entre Rentabilidad y Riesgo en la Planeación de las Finanzas Personales. Retrieved from Scielo: Formación Universitaria: https://www.scielo.cl/scielo.php?script=sci_arttext\&pid=S0718-50062018000600041\&lang=pt

Wu, J., \& Ge, X. (2012). Optimization Research of Generation Investment Based on Linear Programming Model. Physics Procedia, 1400-1405.

Youngdahl, W. E., \& Hunsaker, B. T. (2020). CODA Coffee and Bext360 Supply Chain: Machine Vision, AI, IoT, and Blockchain. Phoenix, Arizona, USA: Thunderbird School of Global Management.

Zavala, J., \& García, D. (26 de Junio de 2009). Selección de una Cartera de Inversión en la Bolsa Mexicana de Valores por Medio de un Método de Programación Lineal. Programación Matemática y Software, 130-151.

\section{Anexos}

Tabla 12.-Interacción 5: La variable que sale de la base es P8 y la que entra es P4.

\begin{tabular}{|c|c|c|c|c|c|c|c|c|c|c|c|c|c|c|c|c|c|}
\hline Tabla 5 & & & 0.35 & 0.48 & 0.1 & 0.21 & 0.69 & 0.12 & 0 & 0 & 0 & 0 & 0 & 0 & O & O & 0 \\
\hline Base & Cb & P0 & P1 & P2 & P3 & P4 & P5 & P6 & P7 & P8 & P9 & P10 & P11 & P12 & P13 & P14 & P15 \\
\hline P15 & 0 & 3000 & 0 & 0 & 1 & -1 & 0 & 1 & 1 & 0 & 0 & 0 & -1 & 0 & 0 & 2 & 1 \\
\hline P8 & 0 & 23000 & 0 & 0 & -1 & 1.5 & 0 & -1 & -1.5 & 1 & 0 & 0 & 1 & 0 & 0 & -2.5 & 0 \\
\hline P9 & 0 & 10000 & 0 & 0 & 1 & 0 & 0 & 0 & 0 & 0 & 1 & 0 & 0 & 0 & 0 & 0 & 0 \\
\hline P10 & 0 & 30000 & 0 & 0 & 0 & 1 & 0 & 0 & 0 & 0 & 0 & 1 & 0 & 0 & 0 & 0 & 0 \\
\hline P1 & 0.35 & 38000 & 1 & 0 & 0 & 0 & 0 & 0 & 1 & 0 & 0 & 0 & 0 & 0 & 0 & 0 & 0 \\
\hline P12 & 0 & 12000 & 0 & 0 & 0 & 0 & 0 & 1 & 0 & 0 & 0 & 0 & 0 & 1 & 0 & 0 & 0 \\
\hline P13 & 0 & 28500 & 0 & 0 & 1 & -0.75 & 0 & 1 & 0.75 & 0 & 0 & 0 & 0 & 0 & 1 & 1.75 & 0 \\
\hline P2 & 0.48 & 22000 & 0 & 1 & 1 & -1.5 & 0 & 1 & 1.5 & 0 & 0 & 0 & -1 & 0 & 0 & 2.5 & 0 \\
\hline P5 & 0.69 & 35000 & 0 & 0 & 0 & 0 & 1 & 0 & 0 & 0 & 0 & 0 & 1 & 0 & 0 & 0 & 0 \\
\hline $\mathbf{Z}$ & & 48010 & 0 & 0 & 0.38 & -0.93 & 0 & 0.36 & 1.07 & 0 & 0 & 0 & 0.21 & 0 & 0 & 1.2 & 0 \\
\hline
\end{tabular}

Tabla 13.-Interacción 6: La variable que sale de la base es P10 y la que entra es P14.

\begin{tabular}{|c|c|c|c|c|c|c|c|c|c|c|c|c|c|c|c|c|c|}
\hline $\begin{array}{l}\text { Tabla } \\
6\end{array}$ & & & 0.35 & 0.48 & 0.1 & 0.21 & 0.69 & 0.12 & 0 & 0 & 0 & 0 & 0 & 0 & 0 & 0 & 0 \\
\hline Base & $\mathbf{C b}$ & P0 & P1 & P2 & P3 & P4 & P5 & P6 & P7 & P8 & P9 & P10 & P11 & P12 & P13 & P14 & P15 \\
\hline P15 & 0 & 18 & 0 & 0 & 0,3 & 0 & 0 & 0.3 & 0 & 0,6 & 0 & 0 & $-0,3$ & 0 & 0 & 0.3 & 1 \\
\hline P4 & 0.21 & 15 & 0 & 0 & $-0,6$ & 1 & 0 & $-0,6$ & -1 & 0,6 & 0 & 0 & 0,6 & 0 & 0 & -16 & 0 \\
\hline P9 & 0 & 10000 & 0 & 0 & 1 & 0 & 0 & 0 & 0 & 0 & 1 & 0 & 0 & 0 & 0 & 0 & 0 \\
\hline P10 & 0 & 15 & 0 & 0 & 0,6 & 0 & 0 & 0,6 & 1 & $-0,6$ & 0 & 1 & $-0,6$ & 0 & 0 & 16 & 0 \\
\hline P1 & 0.35 & 38000 & 1 & 0 & 0 & 0 & 0 & 0 & 1 & 0 & 0 & 0 & 0 & 0 & 0 & 0 & 0 \\
\hline P12 & 0 & 12000 & 0 & 0 & 0 & 0 & 0 & 1 & 0 & 0 & 0 & 0 & 0 & 1 & 0 & 0 & 0 \\
\hline P13 & 0 & 40000 & 0 & 0 & 0.5 & 0 & 0 & 0.5 & 0 & 0.5 & 0 & 0 & 0.5 & 0 & 1 & 0.5 & 0 \\
\hline P2 & 0.48 & 45000 & 0 & 1 & 0 & 0 & 0 & 0 & 0 & 1 & 0 & 0 & 0 & 0 & 0 & 0 & 0 \\
\hline P5 & 0.69 & 35000 & 0 & 0 & 0 & 0 & 1 & 0 & 0 & 0 & 0 & 0 & 1 & 0 & 0 & 0 & 0 \\
\hline $\mathbf{Z}$ & & 62270 & 0 & 0 & $\begin{array}{c}- \\
0.24\end{array}$ & 0 & 0 & $\begin{array}{c}- \\
0.26\end{array}$ & 0.14 & 0.62 & 0 & 0 & 0.83 & 0 & 0 & $\begin{array}{c}- \\
0.35\end{array}$ & 0 \\
\hline
\end{tabular}


Tabla 14.-Interacción 7: La variable que sale de la base es P12 y la que entra es P6.

\begin{tabular}{|c|c|c|c|c|c|c|c|c|c|c|c|c|c|c|c|c|c|}
\hline $\begin{array}{c}\text { Tabla } \\
7\end{array}$ & & & 0.35 & 0.48 & 0.1 & 0.21 & 0.69 & 0.12 & $\mathbf{0}$ & 0 & 0 & 0 & O & O & 0 & 0 & 0 \\
\hline Base & Cb & P0 & P1 & P2 & P3 & P4 & P5 & P6 & P7 & P8 & P9 & P10 & P11 & P12 & P13 & P14 & P15 \\
\hline P15 & 0 & 15400 & 0 & 0 & 0.2 & 0 & 0 & 0.2 & -0.2 & 0.8 & 0 & -0.2 & -0.2 & 0 & 0 & 0 & 1 \\
\hline P4 & 0.21 & 30000 & 0 & 0 & 0 & 1 & 0 & 0 & 0 & 0 & 0 & 1 & 0 & 0 & 0 & 0 & 0 \\
\hline P9 & 0 & 10000 & 0 & 0 & 1 & 0 & 0 & 0 & 0 & 0 & 1 & 0 & 0 & 0 & 0 & 0 & 0 \\
\hline P14 & 0 & 8800 & 0 & 0 & 0.4 & 0 & 0 & 0.4 & 0.6 & -0.4 & 0 & 0.6 & -0.4 & 0 & 0 & 1 & 0 \\
\hline P1 & 0.35 & 38000 & 1 & 0 & 0 & 0 & 0 & 0 & 1 & 0 & 0 & 0 & 0 & 0 & 0 & 0 & 0 \\
\hline P12 & 0 & 12000 & 0 & 0 & 0 & 0 & 0 & 1 & 0 & 0 & 0 & 0 & 0 & 1 & 0 & 0 & 0 \\
\hline P13 & 0 & 35600 & 0 & 0 & 0.3 & 0 & 0 & 0.3 & -0.3 & 0.7 & 0 & -0.3 & 0.7 & 0 & 1 & 0 & 0 \\
\hline P2 & 0.48 & 45000 & 0 & 1 & 0 & 0 & 0 & 0 & 0 & 1 & 0 & 0 & 0 & 0 & 0 & 0 & 0 \\
\hline P5 & 0.69 & 35000 & 0 & 0 & 0 & 0 & 1 & 0 & 0 & 0 & 0 & 0 & 1 & 0 & 0 & 0 & 0 \\
\hline $\mathrm{Z}$ & & 65350 & 0 & 0 & -0.1 & 0 & 0 & $\begin{array}{c}- \\
0.12\end{array}$ & 0.35 & 0.48 & 0 & 0.21 & 0.69 & 0 & 0 & 0 & 0 \\
\hline
\end{tabular}

Tabla 15.-Interacción 8: La variable que sale de la base es P14 y la que entra es P3.

\begin{tabular}{|c|c|c|c|c|c|c|c|c|c|c|c|c|c|c|c|c|c|}
\hline $\begin{array}{c}\text { Tabla } \\
8\end{array}$ & & & 0.35 & 0.48 & 0.1 & 0.21 & 0.69 & 0.12 & 0 & 0 & 0 & 0 & $\mathbf{0}$ & 0 & 0 & 0 & 0 \\
\hline Base & $\mathbf{C b}$ & P0 & P1 & $\mathbf{P 2}$ & P3 & P4 & P5 & P6 & P7 & P8 & P9 & P10 & P11 & P12 & P13 & P14 & P15 \\
\hline P15 & 0 & 13000 & 0 & 0 & 0.2 & 0 & 0 & 0 & -0.2 & 0.8 & 0 & -0.2 & -0.2 & -0.2 & 0 & 0 & 1 \\
\hline P4 & 0.21 & 30000 & 0 & 0 & 0 & 1 & 0 & 0 & 0 & 0 & 0 & 1 & 0 & 0 & 0 & 0 & 0 \\
\hline P9 & 0 & 10000 & 0 & 0 & 1 & 0 & 0 & 0 & 0 & 0 & 1 & 0 & 0 & 0 & 0 & 0 & 0 \\
\hline P14 & 0 & 4000 & 0 & 0 & 0.4 & 0 & 0 & 0 & 0.6 & -0.4 & 0 & 0.6 & -0.4 & -0.4 & 0 & 1 & 0 \\
\hline P1 & 0.35 & 38000 & 1 & 0 & 0 & 0 & 0 & 0 & 1 & 0 & 0 & 0 & 0 & 0 & 0 & 0 & 0 \\
\hline P6 & 0.12 & 12000 & 0 & 0 & 0 & 0 & 0 & 1 & 0 & 0 & 0 & 0 & 0 & 1 & 0 & 0 & 0 \\
\hline P13 & 0 & 32000 & 0 & 0 & 0.3 & 0 & 0 & 0 & -0.3 & 0.7 & 0 & -0.3 & 0.7 & -0.3 & 1 & 0 & 0 \\
\hline P2 & 0.48 & 45000 & 0 & 1 & 0 & 0 & 0 & 0 & 0 & 1 & 0 & 0 & 0 & 0 & 0 & 0 & 0 \\
\hline P5 & 0.69 & 35000 & 0 & 0 & 0 & 0 & 1 & 0 & 0 & 0 & 0 & 0 & 1 & 0 & 0 & 0 & 0 \\
\hline $\mathbf{Z}$ & & 66790 & 0 & 0 & $\begin{array}{c}- \\
0.1\end{array}$ & 0 & 0 & 0 & 0.35 & 0.48 & 0 & 0.21 & 0.69 & 0.12 & 0 & 0 & 0 \\
\hline
\end{tabular}

